Classification

Physics Abstracts

$61.30 \mathrm{E}-64.70 \mathrm{M}$

\title{
DSC and microscopic investigations on HOBACPC
}

\author{
S. C. Jain $\left(^{*}\right)$ and J. Wahl \\ Physikalisches Institut der Universität Münster, Domagkstr. 75, D4400 Münster, FRG
}

(Reçu le 21 juillet 1982, révisé le 14 décembre, accepté le 10 janvier 1983)

\begin{abstract}
Résumé. - Nous présentons des études calorimétriques (DSC) détaillées et des études de texture en phases solide-cristalline et liquide-cristalline sur HOBACPC. A l'aide des études en DSC nous avons mis en évidence l'existence de deux phases solide-cristallines (SC) dans ce composé. L'existence et la prépondérance de ces phases solide-cristallines dépendent de façon critique du passé thermique de l'échantillon. La dégradation thermique du matériau dans l'air et dans l'azote est également étudiée.
\end{abstract}

\begin{abstract}
Detailed DSC and textural studies on HOBACPC in solid and liquid crystalline phases are presented. DSC studies have shown the existence of two solid crystalline (SC) phases in this compound. The existence and the preponderance of these SC phases depend critically on the thermal history of the sample. The thermal degradation of the material in air and in nitrogen atmospheres is reported.
\end{abstract}

HOBACPC (p-hexyloxybenzylidene-p'-amino-2-chloropropyl-cinnamate) is a widely studied ferroelectric liquid crystalline material [1-7]. It has much larger spontaneous polarization than many other ferroelectric liquid crystals. The first reported thermal phase diagram of this compound was as follow [1] :

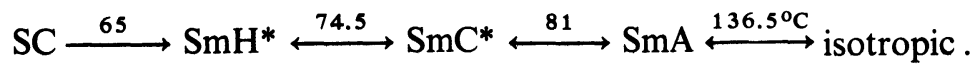

However, in other papers one always finds some difference in these transition temperatures $[1-5,7]$, which is mainly due to the poor chemical stability of this material. There is also evidence that there are more smectic phases [3] than were earlier reported. We present below our detailed DSC and microscopic studies to elucidate on the thermal phase diagram and its modification in thermally degraded samples.

The calorimetric investigations were done on a differential scanning calorimeter, PerkinElmer model DSC2. Figure 1 shows a typical DSC trace of HOBACPC for a heating cycle. On comparing these transition peaks with those of reference [3], it is easy to conclude that the $60,67.5,73.4,80$ and $135.8^{\circ} \mathrm{C}$ peaks should correspond to the SC-SmIV, SmIV-SmIII, SmIII$\mathrm{SmC}^{*}, \mathrm{SmC}^{*}-\mathrm{SmA}$ and SmA-isotropic phase transitions respectively. The SmIV phase always

$\left(^{*}\right)$ Permanent address : National Physical Laboratory, Hill Side Road, New Delhi - 110012, India. 


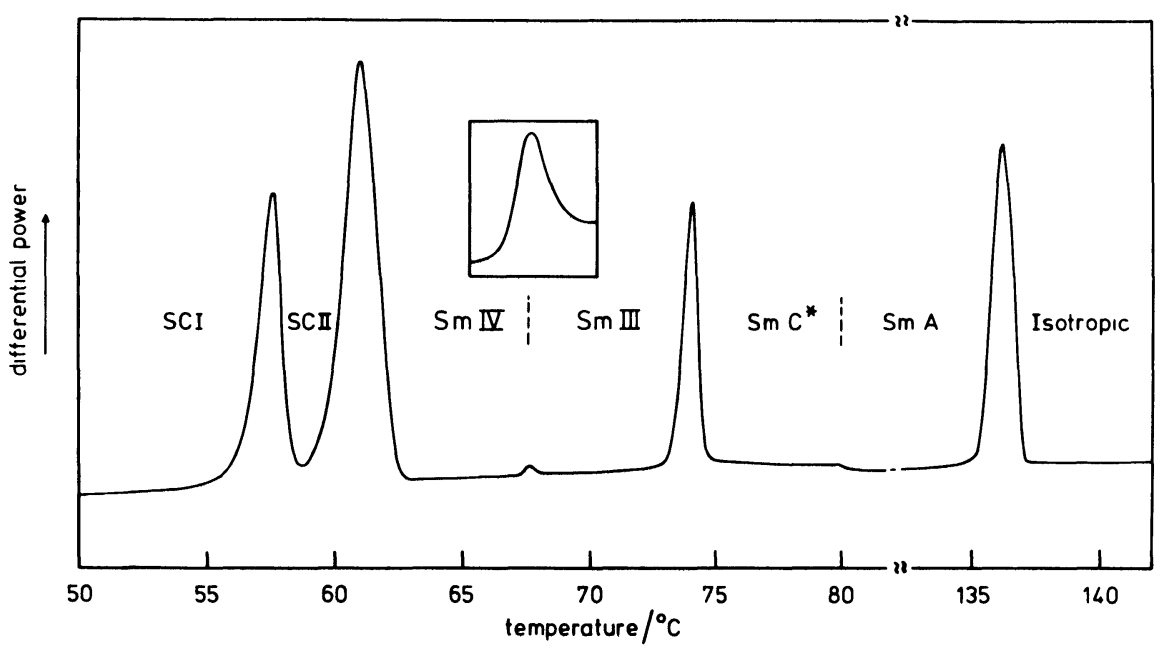

Fig. 1. - DSC trace of HOBACPC during heating cycle (heating rate $2.5^{\circ} \mathrm{C} / \mathrm{min}$.). Inset shows the DSC trace with increased sensitivity.

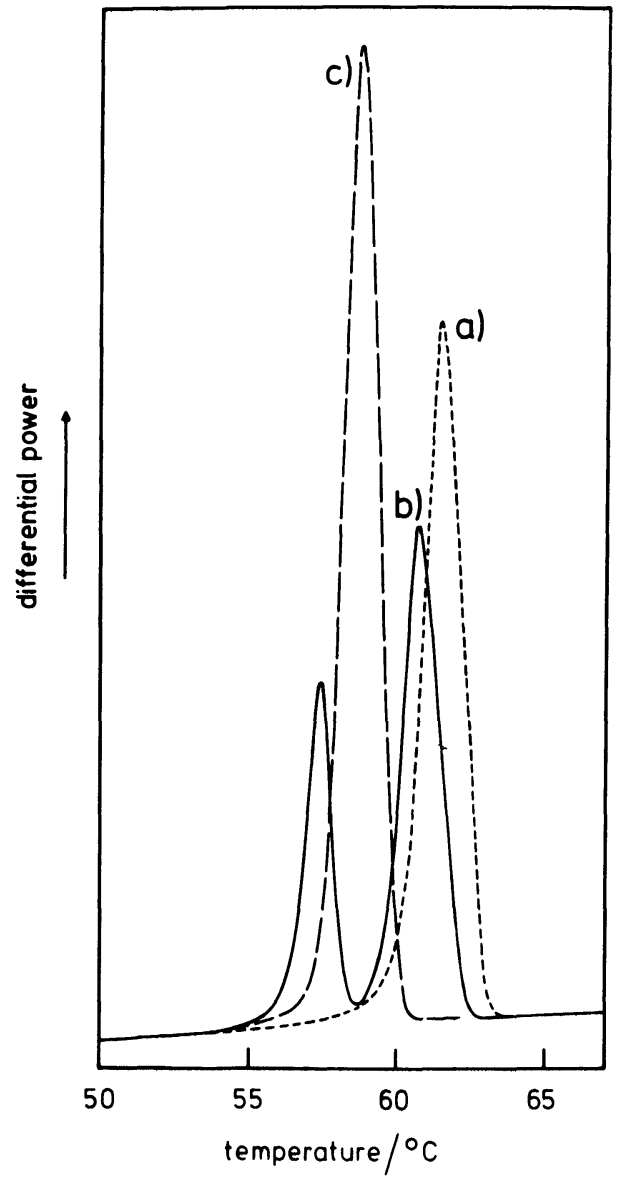

Fig. 2. - A set of DSC traces during heating cycle of the solid forms : $a$ ) obtained by slow cooling; cooling rate $0.65^{\circ} \mathrm{C} / \mathrm{min}$; $b$ ) obtained by rapid cooling; cooling rate $80^{\circ} \mathrm{C} / \mathrm{min}$.; c) obtained by slow cooling, and left one night at room temperature. 
shows a large range of supercooling depending on the cooling rate. In general, the crystallization occurs at around room temperature. It is established through our DSC studies that the $57.2^{\circ} \mathrm{C}$ peak in figure 1 corresponds to another SC form of this compound. Figure 2 shows a set of DSC traces during heating of the SC forms obtained under different cooling conditions. Trace $2 a$ corresponds to the melting of the SC form obtained by very slow cooling of the SmIV phase. It is clear that there is only one solid phase (hereinafter called SCII) which melts at $60{ }^{\circ} \mathrm{C}$ into the SmIV phase. Trace $2 b$ corresponds to the melting of the SC form obtained by fast cooling. One observes two transition peaks at $57.2^{\circ} \mathrm{C}$ and $60^{\circ} \mathrm{C}$ as is also shown in figure 1 . The magnitude of these peaks increases and decreases respectively with increasing cooling rate $\left({ }^{1}\right)$. When the SCII phase, as obtained by very slow cooling, is left one night at room temperature, one observes on heating only one peak at $57.2^{\circ} \mathrm{C}$ (Fig. $2 c$ ). It is very likely that the SCII phase has transformed itself into another stable solid phase (SCI) which melts at $57.2^{\circ} \mathrm{C}$ into the SmIV phase. The different amplitudes of the $57.2^{\circ} \mathrm{C}$ peak represent the partial formation of the SCI phase. When the melt is rapidly cooled there is perhaps no time for the melt to get completely into the SCII phase and part of the melt goes directly into the SCI phase. Such a hypothesis would also explain the observed dependence of the $57.2^{\circ} \mathrm{C}$ and $60^{\circ} \mathrm{C}$ peaks on the cooling rate. The melting behaviour of the SCI and SCII phases can be explained thermodynamically in a way similar to that for another compound studied by Neff et al. [8]. A transition from the SCI to the SCII phase could neither be seen by DSC (Fig. 2c) nor optically. Such a behaviour suggests that the SCI phase gets superheated [8] and goes directly into the SmIV phase.

The enthalpies $(\Delta H)$ associated with the various transitions are :

$\begin{array}{cccccc}\text { SCI-SmIV } & \text { SCII-SmIV } & \text { SmIV-SmIII } & \text { SmIII-SmC* } & \text { SmC*-SmA } & \text { SmA-isotropic } \\ 18.2 & 13.8 & 0.06 & 2.73 & - & 4.89 \mathrm{~kJ} / \text { mole }\end{array}$

We have also carried out DSC studies on thermally degraded samples. It is found that in general the thermal stability of HOBACPC is extremely poor. The various transition temperatures get considerably lowered when the sample is kept in the isotropic phase in air for some minutes only. Typically, when the isotropic to SmA transition temperature had come down to $134.5^{\circ} \mathrm{C}$, the other liquid crystalline transition temperatures shifted down to 77.3, 71.6 and $63.2^{\circ} \mathrm{C}$ respectively. It is observed microscopically that also the application of electric fields, which have been very commonly used to obtain mono-domain samples, lowers the various transition temperatures very considerably. With decreasing SmA-SmC* transition temperature the range of the $\mathrm{SmC}^{*}$ phase is strongly affected. In cases when the SmA-SmC* transition temperature had come down to around $70^{\circ} \mathrm{C}$ (which is not uncommon) the range of the $\mathrm{SmC}^{*}$ phase was reduced to 2 to $3^{\circ} \mathrm{C}$ only. When the isotropic to SmA transition temperature was below $120^{\circ} \mathrm{C}$, no sharp transitions between the various smectic phases could be seen. However, in samples kept in the isotropic phase in dry nitrogen atmosphere degradation is not as rapid. In samples kept at $150^{\circ} \mathrm{C}$ for about $45 \mathrm{~min}$. the isotropic to $\mathrm{SmA}$ transition temperature decreased from $135.8^{\circ} \mathrm{C}$ to $128^{\circ} \mathrm{C}$, whereas the other transition temperatures were not significantly lowered. These results helped us greatly in identifying microscopically the temperature ranges of the various smectic phases in HOBACPC.

Microscopic investigations were carried out in detail to study the textural changes in going through the different smectic phases. The observed changes are different in thick and thin samples $(0.5$ to $120 \mu \mathrm{m})$. With no special boundary treatment to the containing glass plates, the SmA phase, in general, shows a focal conic fan texture (Fig. $3 a$ ). One can also obtain a good homeotropic texture in this phase by a suitable surface treatment and/or by slight shearing of the glass

$\left({ }^{1}\right)$ These two peaks are shifted slightly on the temperature scale with respect to the position of the single peaks obtained when only one solid phase is present. This shift has been neglegted in assigning the positions of the two peaks for the mixed crystalline states. 

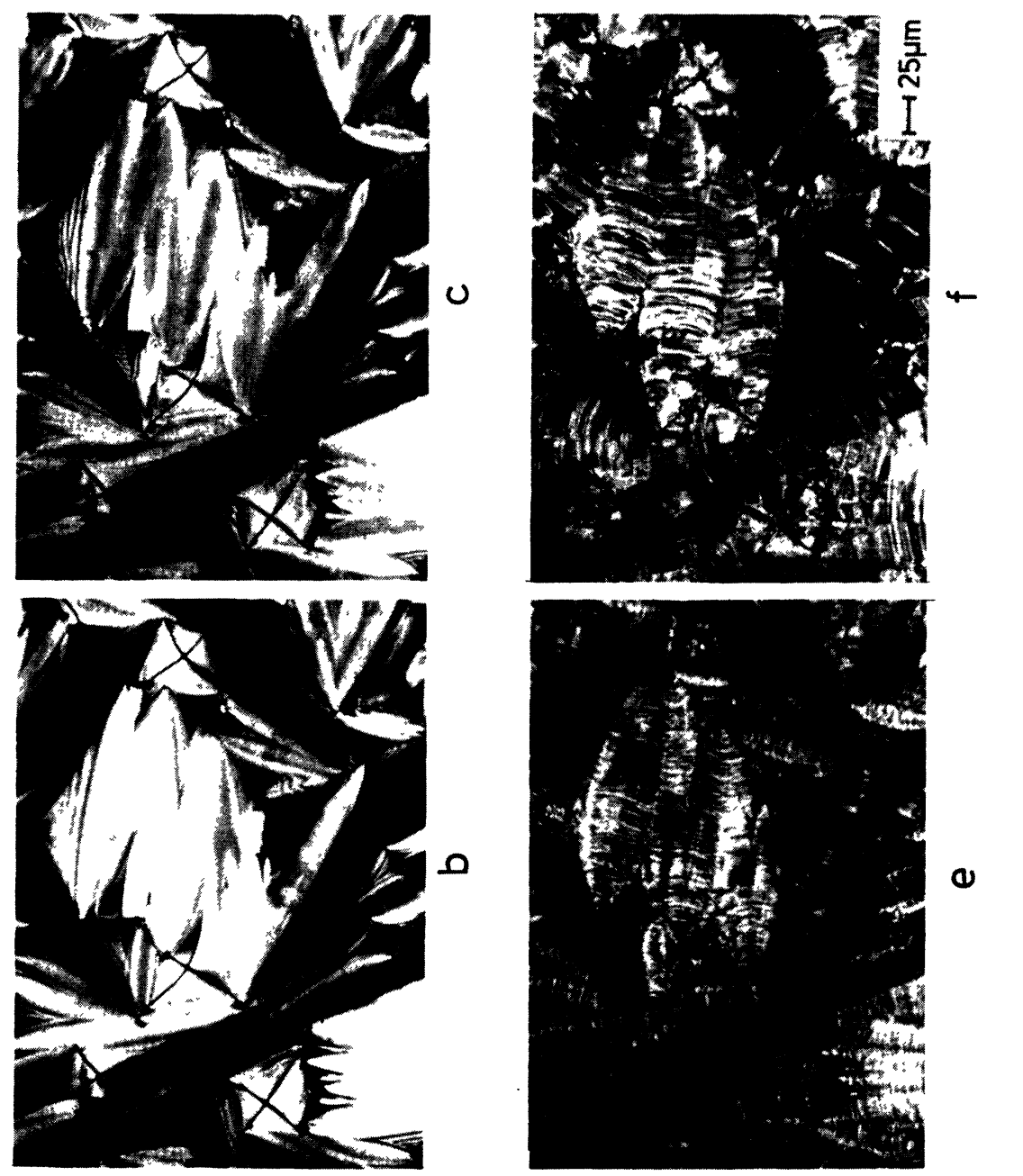

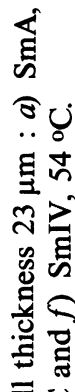

ఫิ

ชิ

岂

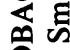

옹

๖ั บ

눈

(1)

홍

ชิ 8

务 ن

늠

冚
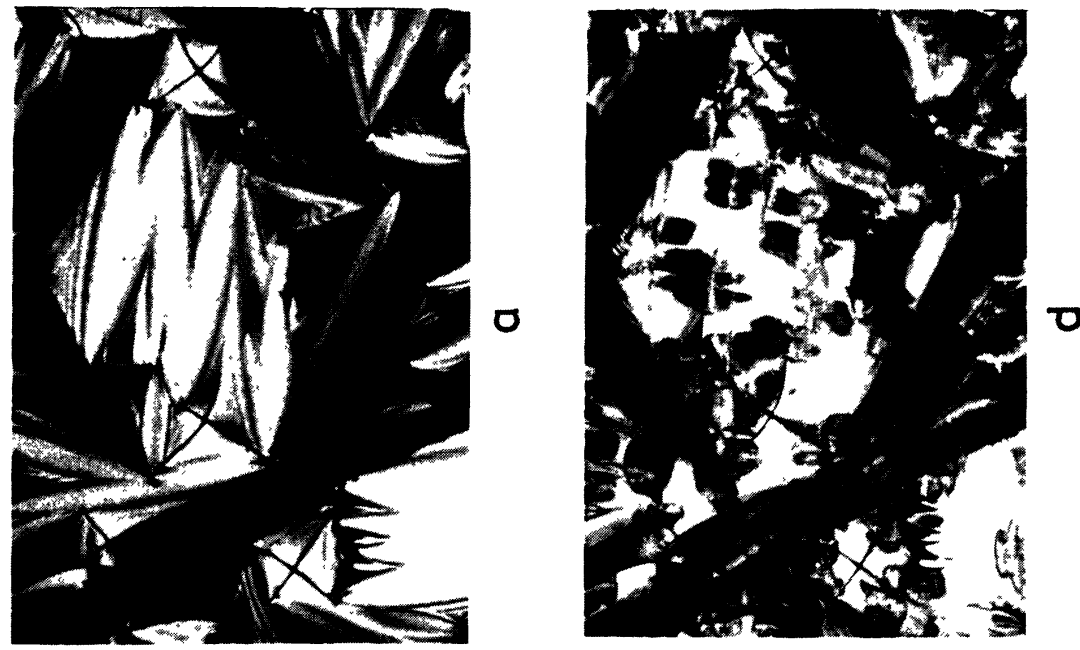

ติ

ลิ

ơ

它

寻

苍

$<$ मี

10

mi்

暲 
plates. Typically, in a sample with an isotropic-SmA transition temperature at $134{ }^{\circ} \mathrm{C}$, on further cooling below $77^{\circ} \mathrm{C}$, the focal conic fan and the homeotropic textures transformed into broken fan (Fig. 3b) and schlieren textures respectively, characteristic of the SmC phase [9]. This transition was marked by an onset of the electro-optic switching [6]. On further cooling a relaxation of the broken fan texture was observed at around $72{ }^{\circ} \mathrm{C}$ (Fig. $3 c$ ). In thick samples $(\gtrsim 25 \mu \mathrm{m})$, this relaxation was accompanied by the appearance of regularly spaced fringes characteristic of a helical structure of the SmC phase [10]. Typically, the spacing between the fringes was around $8 \mu \mathrm{m}$ which is very similar to the pitch value reported by Beresnev et al. [11]. These fringes could be made to disappear on application of a suitable electric field [12]. Invariably, there was observed some difference in temperatures between the onset of the broken fan texture and the appearance of the fringes even in thick samples $(\sim 100 \mu \mathrm{m})$. But this difference was much less in very pure samples. It is likely that the wall anchoring effects and impurities strongly suppress the formation of the helical structure. However, the temperature variation behaviour of the pitch was very similar to that reported for other ferroelectric compounds [13].

Just below the SmC*-SmIII phase transition, we observed a broken fan texture (Fig. 3d) which could not be brought into sharp focus. The observed texture was similar to those published by Demus et al. [9] and by Goodby and Gray [14] for the SmF phase on other liquid crystalline materials. It is important to mention that it is very difficult to differentiate between SmF and SmI phases microscopically or even by miscibility studies [15]. One can differentiate between them through X-rays. A comment about the nature of the SmIII phase is made at the end. In the SmIII phase, a few degrees below the SmC*-SmIII transition temperature, there appear regularly spaced dark and bright fringes characteristic of an helical structure. These fringes in thick samples can be best seen only under a specific orientation of the analyser and the polarizer. The relaxation into the helical structure is a slow process and always takes a long time before the structure is fully developed. Figure $4 a$ shows a typical micrograph in the SmIII phase at $67.1^{\circ} \mathrm{C}$ in a $100 \mu \mathrm{m}$ thick cell. These fringes could be made to disappear on application of a suitable dc electric field (Fig. 4b). Typically, the spacing between the fringes was around 2.5 to $3 \mu \mathrm{m}$ and remained nearly constant over the whole range of the SmIII phase $\left({ }^{2}\right)$. To our knowledge, there exists no data regarding the pitch in this phase.

On going from the SmIII phase to the SmIV phase, the textural changes are more subtle. We observed a broken fan texture with fine lines (Fig. 3e) which on subsequent cooling relaxed

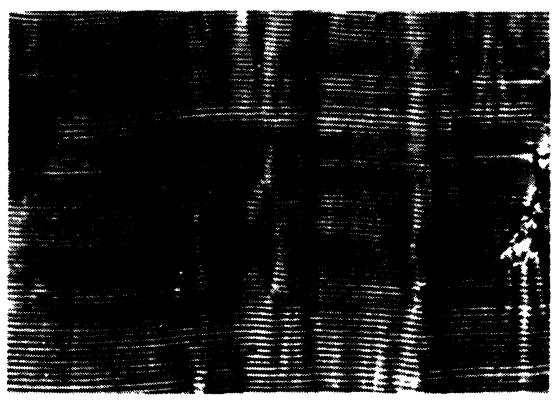

a)

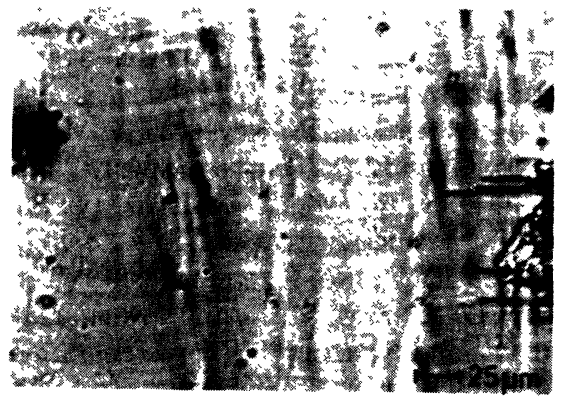

b)

Fig. 4. - Two micrographs of HOBACPC in SmIII phase at $67.1^{\circ} \mathrm{C}$ in $100 \mu \mathrm{m}$ thick cell :a) $U=0 \mathrm{~V}$ and b) $U=50 \mathrm{~V} \mathrm{dc}$.

${ }^{(2)}$ In certain areas, a complete relaxation does not occur even after some hours and it is revealed through unequal spacing between the fringes. 
into a broken fan texture, more typical of the SmH phase [10]. However, on cooling the SmIII phase in a suitable electric field (Fig. $4 b$ ) the changes at the SmIII to SmIV transition were very small. We could not observe any fringes due to an helical structure (both in thick and thin samples). This observation strongly suggests that this phase is non-helical. Such an hypothesis is also supported by X-ray studies of Doucet et al. [3] in this phase which show that there is a strong correlation between the layers. We could observe very good electro-optic bistable switching indicating the ferroelectric nature of this phase. In our preliminary experiments, we could also observe the polarization-reversal oscillogram in this phase. So the SmIV phase would be a pure non-helical ferroelectric liquid crystalline phase.

The nature of the SmIII and SmIV phases as revealed through the X-ray studies of Doucet et al. [3] is characterized by a pseudo-hexagonal order within the layers. The long molecular axes are tilted in a plane parallel to an edge of the pseudo-hexagonal lattice. The two phases mainly differ in that the SmIV phase shows a three dimensional order while the SmIII phase shows a two dimensional order with the layers being almost uncorrelated. Such a SmIII phase has now been termed as SmI [15]. Because of an additional chiral arrangement of the molecules in the successive layers, the SmIII phase should be termed as SmI*. Correspondingly, the SmIV phase should be $\mathrm{SmG}^{\prime}$ or $\mathrm{SmH}^{\prime}$ [15]. But the exact nature of this phase is still not very clear.

Our observation of the non-helical nature of the SmIV phase also suggests that one of the arguments given by DeVries [16] to differentiate between solid crystals and liquid crystals with three dimensional order should be re-examined. DeVries points out that the lattice of a three dimensional smectic phase becomes twisted if the molecules are optically active while the crystal structure of an optically active compound has a single three dimensional lattice. For a twisted smectic structure, he refers to the work of Meyer et al. [12] on the ferroelectric material DOBAMBC. It has been subsequently established that the earlier identified $\mathrm{SmH}^{*}$ phase in this compound is indeed SmI* [17]. So one may ask the question whether a twisted three dimensional smectic phase exists. To our mind, there is still not enough evidence to answer this question in affirmative.

Acknowledgments. - The authors are extremely grateful to Dr. S. T. Lagerwall, Chalmers University of Technology, Sweden, for supplying HOBACPC. One of us (SCJ) is also thankful to DAAD, Bonn, for financial support. We would also like to express our sincere thanks to Dr. Fischer, Pharmazeutische Technologie der Universität Münster, for his help in carrying out the DSC measurements.

\section{References}

[1] Keller, P., Jugé, S., Liébert, L. and Strzelecki, L., C.R. Hebd. Séan. Acad. Sci. 282C (1976) 639.

[2] Martinot-Lagarde, Ph., J. Physique Colloq. 37 (1976) C3-129.

[3] Doucet, J., Keller, P., Levelut, A. M. and Porquet, P., J. Physique 39 (1978) 548.

[4] Durand, G. and Martinot-Lagarde, Ph., Ferroelectrics 24 (1980) 89.

[5] Parmar, D. S. and Martinot-Lagarde, Ph., Ann. Phys. 3 (1978) 275.

[6] Clark, N. A. and Lagerwall, S. T., Appl. Phys. Lett. 36 (1980) 899.

[7] Uemoto, T., Yoshino, K. and InUishi, Y., Mol. Cryst. Liq. Cryst. 67 (1981) 137.

[8] NefF, V. D., Chang, M. K. and Fishel, D. L., Mol. Cryst. Liq. Cryst. 17 (1972) 369.

[9] Demus, D. and Richter, L., Textures of Liquid Crystals (Verlag Chemie, Weinheim, New York) 1978.

[10] Keller, P., Liébert, L. and Strzelecki, L., J. Physique Colloq. 37 (1976) C3-27.

[11] Beresnev, L. A., Balkalov, V. A., Blinov, L. M., Pozhidaev, E. P. and Purvanetskas, G. V., JETP Lett. 33 (1981) 536.

[12] Meyer, R. B., Liébert, L., Strzelecki, L. and Keller, P., J. Physique-Lett. 37 (1976) L-69.

[13] Kondo, K., Takezeo, H., Fukuda, A. and Kuze, E., Jpn J. Appl. Phys. 21 (1982) 224.

[14] Goodby, J. W. and Gray, G. W., Mol. Cryst. Liq. Cryst. 41 (1978) 183.

[15] Gane, P. A. C., Leadbetter, A. J. and Wrighton, P. G., Mol. Cryst. Liq. Cryst. 66 (1981) 247.

[16] DeVries, A., Mol. Cryst. Liq. Cryst. Lett. 49 (1978) 19.

[17] Guillon, D., Stamatoff, J. and Cladis, P. E., J. Chem. Phys. 76 (1982) 2056. 